\title{
Neuromotor development and visual acuity in premature infants submitted to early visuo-motor stimulation
}

\author{
Carla Mazzitelli ${ }^{1}$, Marcelo Fernandes Costa $^{1}$, Solange Rios Salomão ${ }^{2}$, Filomena Maria Buosi De \\ Haro $^{1}$, Adriana Berezovsky ${ }^{1}$, Odete Fernandes Durigon ${ }^{1}$ and Dora Fix Ventura ${ }^{1}$ \\ 1-Universidade de São Paulo, Brazil. \\ 2- Universidade Federal de São Paulo, Brazil.
}

\begin{abstract}
To investigate the effects of early visual stimulation on the development of tonus and posture control and of grating visual acuity of preterm infants, eight infants received visual stimulation that induced head motion during the first 6 months of life in weekly sessions. Their performance was compared with that of unstimulated preterm infants of same age $(n=6)$. Both groups were evaluated in the developmental scale of Gesell and had their grating visual acuity measured by swept visually evoked potentials (sweep-VEP). Visuomotor performance and adoption and maintenance of postures were significantly better for the stimulated group $(p<.05)$ compared to the unstimulated group. There was no statistical difference between the two groups for the development of visual acuity. Visuo-motor and postural development improved with the early visuo-motor stimulation program. The lack of influence of this program in the development of visual acuity may be attributed to differences in the pathways involved in the processing of visual acuity of patterns and in the responses elicited by visuo-motor stimulation. Keywords: psychophysics, preterm infants, sweep VEP, early stimulation, visual acuity, motor development.
\end{abstract}

Received 14 May 2008; received in revised form 29 June 2008; accepted 30 June 2008. Available online 30 June 2008

\section{Introduction}

With the development of neonatology and the consequent reduction in the rate of neonatal death, survival of immaturely born infants has increased. These infants have a greater developmental risk, due in part to having been under intensive care, which often entails inadequate stimulation (Bennett \& Scott, 1997; Behnke \& Eyler, 1999; Chapiesk \& Evankovich, 1997). Compared to

Carla Mazzitelli, Departamento de Fisioterapia, Fonoaudiologia e Terapia Ocupacional da Faculdade de Medicina da Universidade de São Paulo. Solange Rios Salomão, Departamento de Oftalmologia da Universidade Federal de São Paulo - Escola Paulista de Medicina. Filomena Maria Buosi De Haro, Departamento de Psicologia Experimental do Instituto de Psicologia e Núcleo de Neurociências e Comportamento da Universidade de São Paulo. Adriana Berezovsky, Departamento de Oftalmologia da Universidade Federal de São Paulo - Escola Paulista de Medicina. Odete Fernandes Durigon, Departamento de Fisioterapia, Fonoaudiologia e Terapia Ocupacional da Faculdade de Medicina da Universidade de São Paulo. Dora Fix Ventura, Departamento de Psicologia Experimental do Instituto de Psicologia e Núcleo de Neurociências e Comportamento da Universidade de São Paulo. Correspondence regarding this article should be addressed to Marcelo Fernandes da Costa, Universidade de São Paulo, Departamento de Psicologia Experimental do Instituto de Psicologia e Núcleo de Neurociências e Comportamento da Universidade de São Paulo, Av. Prof. Mello Moraes, 1721, Bloco A sala D-9 São Paulo, SP, Brasil, 05508900. Pnone: +55-11-3091-4444 R:218. Fax: +55-11-3091-4357. E-mail: costamf@usp.br full-term infants, preterms are less alert, active and sociable, differing in visual attention, respiratory pattern, muscle tone and tendinous reflexes. Preterms are at greater risk of reduced rate of physical growth, language development delay, balance and motor coordination deficits (Campbell, 1997).

Prematurity is associated with visual problems such as reduction of visual acuity, strabismus and retinopathy of prematurity (Birch, 2001). There is also the possibility of developing clinical signs of neurological lesions. However, only intense damage is noticeable at birth and during the neonatal period. Milder conditions result in a slower and subtler development (Allen \& Alexander 1997). Therefore, the importance of improving procedures for functional diagnosis in preterm infants and for the corresponding treatment cannot be understated. Such procedures might help prevent future disability and improve the development of these children during the first years of life.

How can development be improved? Since the 1960s, controlled animal studies have demonstrated that an enriched environment may enlarge brain size, increasing the number of neurons, dendrites, synapses and some types of glia, with clear effects on behavior, especially on learning and memory (Diamond, 2001). These findings influenced education and prompted the formulation of early stimulation programs for children in general and specifically for preterm infants.

The "Infant Health and Development Program", consisting of weekly home visits with a program of games 
and activities that the family is instructed to perform with the child, resulted in significant developmental improvement (Resnick, Eyler, Nelson, Eitzman, \& Bucciarelli, 1987). Improvement in physical and mental development in relation to unstimulated infants was reported following the application of a multimodal stimulation program to a group of 104 children using vestibular, visual, auditory, and tactile stimulation within the intensive therapy unit followed by home visits (White-Traut, 2001). Increased alertness and decreased length of hospitalization were the results of another program, which performed auditory, tactile, visual and vestibular interventions in 37 children for 15 minutes, twice daily, 5 days a week, until discharge from the hospital (White-Traut et al., 2002). Another study of preterm infant responses to stationary and rotational visual patterns placed in the incubators in the neonatal intensive care unit showed that visually stimulated infants experienced fewer state changes in the sleep-wakefulness cycle and stronger visual skills (fixation and tracking) than unstimulated infants (Marshall-Baker, Lickliter, \& Cooper, 1998). These findings suggest that visual patterns may promote behavioral organization and visual skills in preterm infants.

Most studies about the effects of early stimulation on development, however, include interventions during the short period after birth, at the hospital. Studies with a planned, controlled, and quantifiable intervention during the first few months of life have not been reported, so the possible effects of a stimulation program during this phase are still unknown.

The purpose of the present study is to determine whether a program of visual stimulation which induces head motion during the first six months of life could influence the development of muscle tonus, posture and grating visual acuity in a small cohort of premature infants.

\section{Method}

\section{Participants}

The subjects were 14 healthy premature infants born in the University Hospital of the University of São Paulo, Brazil. Absence of pathologies at the pediatric clinical exam, that could prevent normal development, was required for inclusion. The parents or adults responsible for each child signed a letter of informed consent approved by the local IRB (Ethical Committee of the University Hospital of the Medical School of University of São Paulo).

The stimulated group $(n=8)$ was composed of babies whose mothers could bring them to the stimulating program. The subjects in this group were submitted to $30 \mathrm{~min}$ sessions of visual stimulation, provided by the presentation of a slowly moving visual target from the right to the left and vice-versa, in the visual horizontal plane at eye level, and along the midline vertical meridian to induce left-right and up-down head motion, including flexion and extension movements. Weekly sessions started in the first month of life and lasted until the sixth month. Each infant received a total of 12 to 15 sessions. Visual stimuli were manually presented by an experimenter and had the objective of attracting the infants' fixation and following of the movement. They were two rubber soccer-type balls (infant toys), of respectively 11 and $5 \mathrm{~cm}$ in diameter, the first with a black and white pattern and the second with an equivalent pink and blue pattern, presented at a distance of about $30 \mathrm{~cm}$, comprising visual angles of $20^{\circ}$ and $9.4^{\circ}$, respectively. This program, proposed by Durigon and collaborators consists of presenting the stimulation series in the dorsal and ventral lying postures as well as sitting. The infant was positioned alternately in each posture for about 5 to $10 \mathrm{~min}$.

The unstimulated group was composed of 6 healthy preterm infants that were not submitted to the stimulation program. Unfortunately, the small number of babies and practical difficulties in bringing the babies to the stimulation program prevented randomization in assigning them to the different groups. The stimulation program was performed in the Department of Physiotherapy, Phonoaudiology and Occupational Therapy of the Medical School of the University of São Paulo.

\section{Motor and visuo-motor assessment}

Both groups were evaluated monthly up to the $4^{\text {th }}$ month of corrected age and then at the $6,8,10$ and $12^{\text {th }}$ months. At these intervals, they were submitted to the neurological exam of the University Hospital of Medical School, and evaluated by Gesell's developmental scale, according to the procedures described in detail by Gesell and Amatruda (1953). This evaluation is based upon the normal development of full-term infants and consists of behavioral tests whose outcome is classified as either pass or fail. In each given test, either inability to perform, or performance in a pattern that differs from that described by Gesell and Amatruda is rated as failure. The tests that evaluate visuomotor abilities are related to the infants' capacity to focus and follow a visual stimulus with the movement of the eyes, head and arm in the direction of the object, according to the age. The tests that evaluate posture examine the capacity of the child to maintain different postures (lying in supine or prone positions, sitting, kneeling and standing) according to age.

\section{Visual acuity assessment}

All infants were submitted to at least three measurements of grating visual acuity, at $6,17,26$, and 48 weeks of corrected age, tested using the NuDiva version of the sweep visual evoked potential (sVEP) system. The sVEP measurements were performed at the Vision Lab at the Institute of Psychology of the University of São Paulo. The stimuli were black and white vertical squarewave reversal gratings produced on a high-resolution video monitor (Dotronix Model EM2400-D788) with 80\% contrast and mean luminance of $160 \mathrm{~cd} / \mathrm{m}^{2}$. Ten increasing spatial frequencies were swept, reversing at $6 \mathrm{~Hz}$, with the frequency range at any value between .25 and 40 cycles per degree. Testing distance was 30,50 or $100 \mathrm{~cm}$ according to the age of the child. Recordings were obtained with EEG electrodes (Grass gold disk - E6GH) at $\mathrm{O} 1$ and $\mathrm{O} 2$, with the 
reference electrode $(\mathrm{Oz}) 1 \mathrm{~cm}$ above inion on the midline and a ground electrode $2-3 \mathrm{~cm}$ above $\mathrm{Oz}$. The signal was amplified by a Neurodata Acquisition System (Grass mod. 12C-4-23) and processed online by the NuDiva system, which used a discrete Fourier transform to measure amplitude and phase of the second harmonic (at $12 \mathrm{~Hz}$ ) of the grating reversal rate $(6 \mathrm{~Hz})$.

The test was performed in a darkened room with fixation maintained with small toys dangled in front of the video monitor to draw the infant's attention. Grating acuities were determined as the average of three to 12 repetitions of the sweep, calculated with the requirement of a signal to noise ratio of 3 at peak amplitude, for both channels. Results were expressed in log MAR (minimum angle of resolution) of the threshold of the best eye and compared with normative data (Salomão \& Birch, 1996). The sweep VEP methodology has been successfully used to estimate visual functions like contrast sensitivity in full-term and preterm infants (Oliveira, Costa, de Souza, \& Ventura, 2004) and the visual acuity in children with neurological alterations like spastic cerebral palsy (Costa, Salomão, Berezovsky, Haro, \& Ventura, 2004).

\section{Results}

The stimulation program and the sweep VEP were successfully performed in all infants of the stimulated group. The sweep VEP was also performed successfully in all infants of the unstimulated group. The results of the evaluation with the Gesell scale were analyzed using the Student $t$-test to compare the performances of the stimulated and unstimulated groups at each of the tests, at the different ages. The percentage of tests in which the stimulated group's performance was statistically higher ( $p$ $<.05)$ than the unstimulated group is presented in Figure 1. There was significant improvement in $67 \%(S D=25 \%)$ of the visuo-motor abilities and $53 \%(S D=22 \%)$ of the

\section{Gesell Scale}

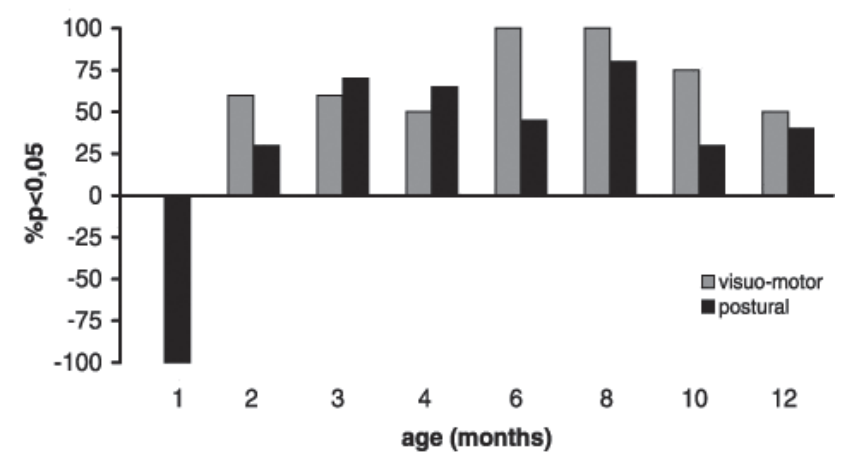

Figure 1. Percentage of infants of the stimulated group whose scores in tests of visuo-motor abilities in the Gesell Scale were statistically different $(p<.05)$ from those of the unstimulated group. The tests that evaluate posture examine the capacity of the child to maintain different postures (lying in supine or prone positions, sitting, kneeling and standing) according to age. postural functions in the stimulated group relative to the unstimulated group.

The results obtained from the analysis of the sweep VEP at corresponding ages are shown in Figure 2. The grating visual acuity values are within the normal limits for the age and the development of the visual function is similar to that of full-term infants. There were no significant differences between the visual acuities estimated by the sweep VEP between the preterm infants of the stimulated group and those of the unstimulated group (Mann Whitney $\mathrm{U}$ test, $p>.05$ ). Visual acuities measured with the same technique in full-term infants and reproduced in Figure 2 for comparison.

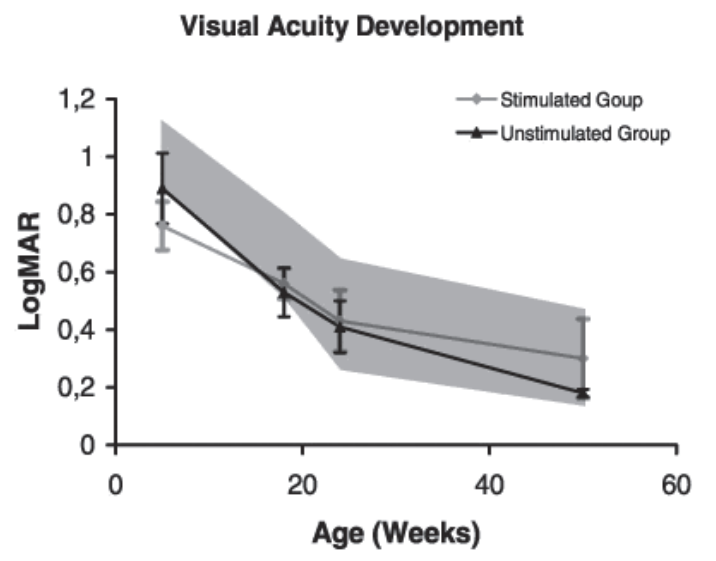

Figure 2. Visual acuities measured by the sweep VEP at different ages, for the stimulated and unstimulated groups of infants. Visual acuities for full-term infants (mean plus lower tolerance limit) measured by Salomão and Birch (1196) are reproduced as a gray band for comparison.

\section{Discussion}

The stimulation program was effective in improving the development of visuo-motor and postural functions. In the stimulation program the infant was induced to actively fixate and track the object through the visual field, implying in simultaneous vestibular activation and stimulation of central areas related to visuo-motor control. The resulting developmental changes measured suggest that this program acted to improve aspects of the motor development involved in visuo-motor abilities and in the ability to keep posture and tonus. The improvements were recorded not only during the six months of application of the program but also in the following six months, up to 12 months of age. The largest effect of the program was seen in the first 8 months. From that age on the environment in which the child develops is probably more relevant for the motor acquisitions than those stimulated through the program. These results agree with other studies that used different stimulation programs (Diamond, 2001, Resnick et al., 1987, White-Traut, 2001, White-Traut et al., 2002).

However, some limitations of this study should be considered. One is due to the fact that the infants could 
not be randomized to the two groups since not all parents could bring them to the stimulating sessions. Another one is the fact that the same experimenter performed the stimulation program and the tests; therefore, she was not blind to which group the infant being tested belonged to. A further limitation was the fact that the parents knew that the child was in a stimulation group, since they brought the infant to the session, which might have prompted them to further stimulate the infant at home. Finally, the experimental group was formed by babies whose parents were willing and could bring them to the sessions, while for the control group this was not possible. These differences might reflect other family variables that could have interfered with the results. Therefore, further studies with a stricter control of the variables that were pointed out must be performed before definite conclusions about the improvement in visuo-motor and postural performance exclusively to the stimulation program. Nevertheless, the fact that this improved performance has also been found by other authors suggests that the stimulation program is beneficial (Diamond, 2001, Resnick et al., 1987, White-Traut, 2001, White-Traut et al., 2002).

In contrast with the visuo-motor and postural performances, visual acuities of preterm infants from the unstimulated and stimulated groups had a similar improvement with age, with no statistical difference between groups (see Figure 2). Both sets of results fell within the age norms obtained with full-term infants (Salomão \& Birch, 1996).

The absence of differences in the visual acuities of fullterm and preterm infants was also reported by Birch and Spencer (1991), who found that neither the age at testing nor the gestational age at birth influenced the development of grating acuity during the first year of life of healthy preterm infants, in comparison with full-term controls. There were also no differences in contrast sensitivities measured by the same method in full-term and healthy preterm infants (Oliveira, et al., 2004). However, using behavioral tests, Baraldi, Ferrari, Fonda, \& Penne (1981) found that the visual acuity of healthy preterms is a little better than that of full-term infants at the corrected age.

Our results suggest that the longer period of visual experience in preterm infants does not affect the capacity of the visual system to resolve spatial stimuli. The experience of the pre-term infants probably affects the synapses of the cortical visual areas that process visual information at higher levels and in the visual association cortex. According to Marian Diamond (2001) the higher cortical areas are more receptive to the environment richness than other cortical areas. These differences might explain the higher visual acuity found on preterm infants by means of behavioral measures by Baraldi et al. (1981), which were not confirmed in physiological measurements by Oliveira et al. (2004)

Experience during the critical period, a period after the birth in which visual system is more sucptible to changes, is necessary to guarantee the normal development of the visual pathways and their functions and it is well known that interruptions of sensory experience during this period may lead to impairment of the visual function. In our results we show that the additional experience that the preterm infant has, compared to full-term infants, does not improve visual acuity as measured by VEP, excluding this level of processing from a possible beneficiary of this experience. There is the possibility that differences between preterm and full-term infants can be found in behavioral measurements as shown for visual acuity by Baraldi et al. (1981) and suggested by Diamond's studies (Diamond, 2001).

\section{Effects of early sensory stimulation}

The lack of influence of the stimulation program in the grating visual acuities as opposed to its effect in the visuo-motor and postural behaviors may be understood by an analysis of the neural pathways involved. VEPs reflect primarily functions processed by the parvocellular pathway, while the stimulation program activated mainly the magnocellular pathway and the dorsal stream, given its characteristics of movement and low spatial frequencies, although there were also components of the parvocellular pathway being activated, such as high spatial contrast features (Maunsell, 1992; Rovano \& Virsu, 1979). Another possible explanation is that the difference observed in the stimulated group could reflect the effect of the stimulation program on functions subsequent to that measured in the primary visual cortex by the sweep VEP. Behaviors that depend on good fixation and acuity, such as stimulus tracking or visually guided posture maintenance, could be the main target of the stimulation program. This idea is supported by Diamond's (2001) study which suggests that the areas that are most susceptible to the environmental enrichment are the areas of neural integration. Since the grating acuities are processed by the primary visual area, this would explain why they are not affected by the stimulation program. In conclusion, the results obtained in our study showing an improvement in the postural and visuo-motor functions, with no differences in the visual acuity electrophysiologically measured, could reflect a better use of visual information by the infants that underwent the stimulation program.

\section{Acknowledgements}

Work supported by a graduate fellowship from CAPES to M.F.C. and by FAPESP \#0030/00-98, FINEP \#66.95.0407.00, CNPq \#523303/95-5 grants to D.F.V. Prof. John Manuel de Souza and Claudiel Luiz dos Santos are gratefully acknowledged for general assistance.

\section{References}

Allen, M.C., \& Alexander, G.R. (1997). Using motor milestones as a multistep process to screen preterm infants for Cerebral Palsy. Developmental Medicine and Child Neurology, 39, 12-16. 
Baraldi P., Ferrari F., Fonda S., \& Penne A. (1981). Vision in the neonate (full term and premature): preliminary result of the application of some testing methods. Documenta Ophthalmologica, 51, 101-112.

Behnke, M., \& Eyler, F.D. (1999). Cranial ultrasound abnormalities identified at birth: their relationship to perinatal risk and neurobehavioral outcome. Pediatrics, 103, 103-104.

Bennett, F.C., \& Scott, D. (1997). Long-term perspective on premature infant outcome and contemporary intervention issues. Seminars in Perinatology, 21, 190-201.

Birch, E.E., \& Spencer, R. (1991) Monocular grating acuity of healthy preterm infants. Clinical Visual Science, 6(4), 331-334.

Birch, E.E. (2001). Preterm birth and visual development. Seminars in Neonatology, 6, 487-497.

Campbell, S.K. (1999). The infant at risk for developmental disability. In: Campbell, S.K. Decision making in pediatric physical therapy. New-York: Churchill Livingstone 260-332.

Chapiesk, M.L., \& Evankovich, K.D. (1997). Behavioral effects of prematurity. Seminars in Perinatology, 21, 70-88.

Costa, M.F., Salomão, S.R., Berezovsky, A., Haro, F.M.B., \& Ventura, D.F. (2004). Relationship between vision and motor impairment in children with spastic cerebral palsy: new evidence from electrophysiology. Behavioural Brain Research, 149, 145-150.

Diamond, M.C. (2001). Response of the brain to enrichment. Anais da Academia Brasileira de Ciências, 73(2), 210-220.

Durigon, O.F.S., Mazzitelli, C., Garcia, J.M., Favale, T., \& Maioral, M. (1999). Efficiency of a visual intervention program in premature infants. World Congress of Physical Therapy, Yokohama, Japan, 261-261.

Gesell, A., \& Amatruda, C. (1953). Diagnostico del Desarrollo Normal y Anormal del Niño. Buenos Aires: Medico Paidos.
Marshall-Baker, A., Lickliter, R., \& Cooper, R.P. (1998). Prolonged exposure to a visual patern may promote behavioral organization in preterm infants. Journal of Perinatal and Neonatal Nursing, 12(2), 50-62.

Maunsell, J.H. Functional visual streams. (1992). Current Opinion in Neurobiology, 2, 506-510.

Oliveira, A.G.F., Costa, M.F., de Souza, J.M., \& Ventura, D.F. (2004) Contrast sensitivity threshold measured by sweep-visual evoked potential in term and preterm infants at 3 and 10 months of age. Brazilian Journal of Medical and Biological Research, 37, 1389-1396.

Resnick, M.B., Eyler, F.D., Nelson, R.M., Eitzman, D.V., \& Bucciarelli, R.L. (1987). Developmental intervention for low birth weight infants: Improved Early Developmental Outcome. Pediatrics, 80(1) 68-74.

Rovano, J., \& Virsu, V. (1979). An estimation and application of the human cortical magnification factor. Experimental Brain Research, 37, 495-510.

Salomão, S.R., \& Birch, E.E. (1996). Individual growth curves for sweep-VEP and FPL in the first year of life. Investigative Ophthalmology and Vision Science, 37, S1067.

White-Traut, R.C., Nelson, M.N., Silvestri, J.M., Vasan, U., Littan, S., Meleedy-Rey, P. et al. (2002). Effect of auditory, tactile, visual, and vestibular intervention on length of stay, alertness, and feeding progression in preterm infants. Developmental Medicine and Child Neurology, 44(2), 91-97.

White-Traut, R.C. (2001). One-year outcome of auditory-tactilevisual vestibular intervention in the neonatal intensive care unit: Effects of severe prematurity and central nervous system injury. Journal of Child Neurology, 16(7), 493-498. 
